\title{
Resonant Transmission of Microwaves through a Narrow Metallic Slit
}

\author{
Fuzi Yang and J.R. Sambles \\ Thin Film Photonics, School of Physics, University of Exeter, Exeter EX4 4QL, United Kingdom
}

(Received 12 December 2001; published 22 July 2002)

\begin{abstract}
Strong resonant transmission of microwave radiation through a very narrow (much less than the radiation wavelength) metallic slit is recorded. The results show that a set of resonant self-coupled surface plasmons are excited within the small gap, giving a Fabry-Pérot-like behavior in accord with analytical results published earlier [Y. Takakura, Phys. Rev. Lett. 86, 5601 (2001)]. The metallic slit, formed by two thick metal plates spaced apart by tens of microns, is inserted in a wavelength-sized aperture. On resonance the transmissivity through the metal slit is more than 2 orders of magnitude greater than the radiation impinging on the slit area.
\end{abstract}

PACS numbers: $41.20 . \mathrm{Jb}$

Very recently, remarkable transmission of light via 2D periodic arrays of subwavelength metallic holes in otherwise fully reflecting screens has been reported [1]. The transmitted spectral response exhibits much more transmission than expected for an equivalent set of single holes in an opaque aperture as predicted by standard aperture theory [2]. Apart from its fundamental interest, this extraordinary transmission effect has applications potential which has consequentially stimulated much new work. While discussion still continues about the detailed interpretation [3] of this effect, most agree that the enhanced transmission is related to surface plasmon excitation. There is first diffractive coupling into some form of localized mode at the grating surface, followed by evanescent tunneling through the small holes, followed again by diffractive coupling back to free radiation. While the study by Ebbesen et al. [1] was strictly limited to holes, some model calculations have been undertaken for slits in metal screens [4-8]. In this instance, high transmission is also predicted for a periodic array of subwavelength slits. However, because slits have a very different symmetry to holes, the mechanism for this enhanced transmittance is different, involving propagating, rather than evanescent, modes in the slits. These propagating modes in the narrow slits, for radiation polarized with its $E$ field perpendicular to the slit axis, may be regarded as either waveguide modes or coupled surface plasmons. In order to give a clear physical explanation of the origin of the extraordinary transmission from a metallic slit grating, Takakura [9] analyzed the interaction of TM-polarized waves with a single subwavelength metallic slit. The results show, for a thick enough conducting plate, a series of resonant transmission peaks as a function of incident wavelength. These resonances arise through the setting up of standing waves between the two ends of the slit, Fabry-Pérot-like modes. The "extraordinary transmission" observed in such metallic slit gratings is then the result of excitation of these Fabry-Pérot-like resonances localized in each slit cavity.

In this Letter we experimentally realize transmission through a single narrow metallic slit in a wavelength- width plate. The results confirm the predictions from [9], especially the Fabry-Pérot-like behavior, and show that even a single very narrow slit placed in a metal plate in an absorbing aperture may act as a resonant transmission filter for radiation.

The radiation wavelength used in this experiment is in the microwave region. This choice of microwave radiation is particularly expedient for the following reasons. First, according to [8], the transmission properties of metallic slits with finite conductivity are substantially different from those of perfect conductors, and the condition assumed in [9] is a single narrow slit in a perfect conductor. So, in order to compare the experiments with the predictions from [9], it is appropriate to more closely satisfy the condition of a perfect conductor. In the microwave region metals may be considered as almost perfectly conducting [10]. Second, it is much easier to fabricate and to vary the dimensions of a single slit sample in this long wavelength region.

The experimental arrangement is shown in Fig. 1. The metallic slit sample is made from two $3 \mathrm{~mm}$ wide aluminum plates with two strips of insulating Mylar inserted at
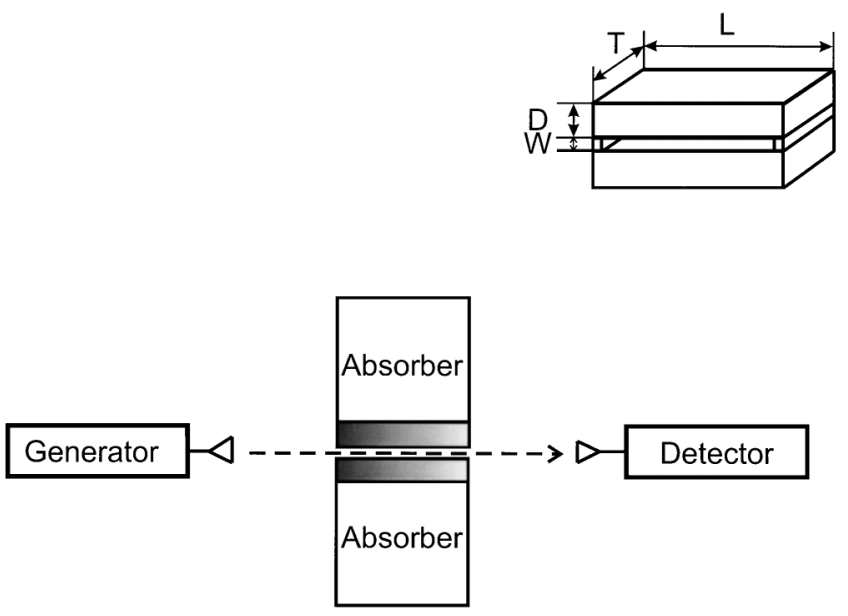

FIG. 1. The experimental setup and sample geometry. 
both short edges of the sample to give a narrow air gap as shown in the corner inset of Fig. 1. This slit is inserted in a wavelength size aperture (6 mm wide) made from highly absorbing material, and the whole assembly is placed between microwave source and detector horns. The source horn is $\sim 1 \mathrm{~m}$ from the front face of the sample to give an essentially plane wave incident. Thereby the incident field intensity distribution at the sample has a uniformity of intensity of better than $10 \%$. In addition, the detection horn is placed about $0.3 \mathrm{~m}$ from the sample, far enough away to be reasonably far field but close enough to give a strong signal. The complete sample is rotated a little such that the incidence angle is $\theta \sim 10.0^{\circ}$ to avoid strong interference effects between the surfaces of the metallic part of the sample and the horns. Only linearly polarized microwaves are incident with the electric vector lying perpendicular to the slit direction; i.e., the incident beam is TM polarized. As expected, no measurable transmission is obtained for radiation polarized along the slit direction. Transmission data were taken as a function of frequency.

A typical set of transmission data, from 40.0 to $75.0 \mathrm{GHz}$, is shown in Fig. 2. For these data the dimensions of the metallic slit are $L=60.0 \mathrm{~mm}, T=28.2 \mathrm{~mm}, D=$ $3.0 \mathrm{~mm}$, and $W=75.0 \mu \mathrm{m}$. The transmission signal is normalized with respect to the signal obtained with an empty aperture in the absorber. From Fig. 2 it is very clear that the transmission through the metallic slit has a Fabry-Pérot-like character. This result coincides with the conclusion of Ref. [9] for which, if $W / \lambda$ ( $\lambda$ is wavelength of the radiation) is small enough, the transmission from a metallic slit will exhibit maxima at frequencies which satisfy the Fabry-Pérot condition. For the present situation the value of $W / \lambda$ is less than 0.02 .

From the theoretical analysis of the single metallic slit [9], there is a fundamental difference between it and a Fabry-Pérot condition. In the slit case additional, wavelength dependent, terms in the denominator cause a shift of the resonant wavelengths, which is expressed as [9]

$$
\lambda_{\text {shift }} / \lambda_{\mathrm{FP}}=2(W / T)\left[\ln \left(\pi W / \lambda_{\mathrm{FP}}\right)-3 / 2\right] /\left\{2(W / T)\left[\ln \left(\pi W / \lambda_{\mathrm{FP}}\right)-1 / 2\right]-\pi\right\},
$$

where $\lambda_{\mathrm{FP}}$ would be the Fabry-Pérot wavelength. For the case here $W / T$ and $W / \lambda_{\mathrm{FP}}$ are very small (of order $10^{-2}-10^{-3}$ ), in which case $\lambda_{\text {shift }} / \lambda_{\mathrm{FP}}$ is positive and also very small. Nevertheless, it should be measurable in the experiment. This $\lambda_{\text {shift }}$ is plotted against $\lambda_{\mathrm{FP}}$ in Fig. 3. The six star symbols on the lower horizontal line indicate the $\lambda_{\mathrm{FP}}$ values calculated according to our experimental geometry, and the six open triangles on the upper, sloped, line are the values of $\lambda_{\text {shift }}$ calculated from Eq. (1). The six filled circles are the experimental data from Fig. 2. It is very clear that even though the wavelength shifts are very small, the relative deviation being less than $1.0 \%$, the experimental results give good agreement with the theoretical prediction.

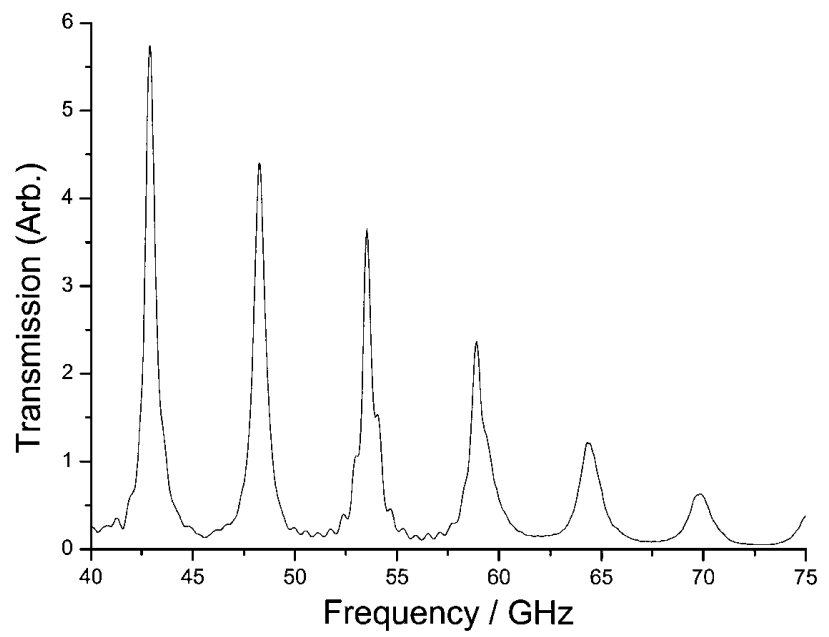

FIG. 2. The transmission spectrum through a single metallic slit.
It is, of course, apparent from Fig. 2 that a very narrow (compared with the wavelength of radiation) metallic slit is definitely not a normal aperture. The transmittance of the single metallic slit of $75 \mu \mathrm{m}$ width is about 6 times that of the empty, $6.075 \mathrm{~mm}$ wide, aperture. If this transmission ratio, shown in Fig. 2, is rescaled to the fraction of surface area occupied by the slit $(1 / 81)$, it gives the effective increased transmittance factors shown as filled circles in Fig. 4. The maximum value is 466 at a wavelength of about $0.70 \mathrm{~cm}$. Given then that the microwave intensity is approximately uniform across the aperture, we deduce that over 400 times as much radiation is transmitted as impinges directly on the area presented by the slit.

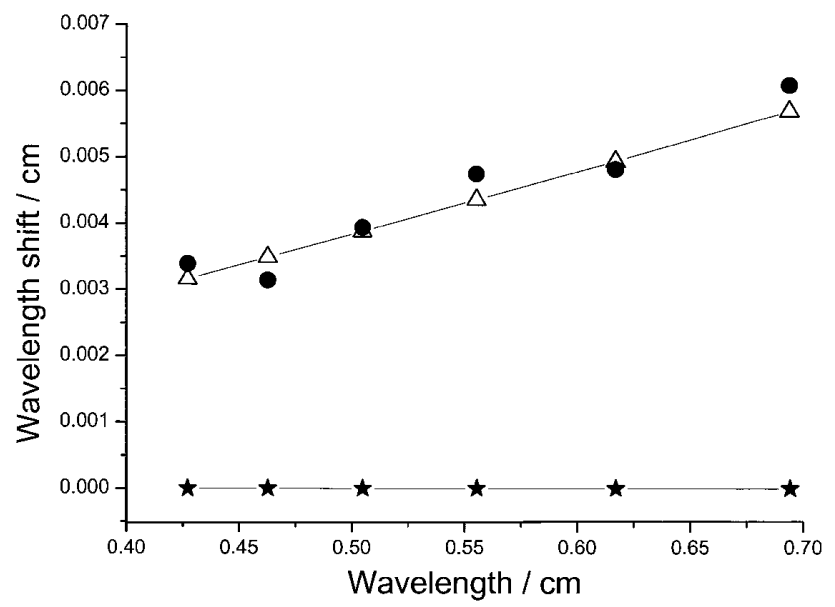

FIG. 3. Theoretical prediction $(\triangle)$ and experimental data $(\bullet)$ of the wavelength shifts from the simple Fabry-Pérot resonance condition. 


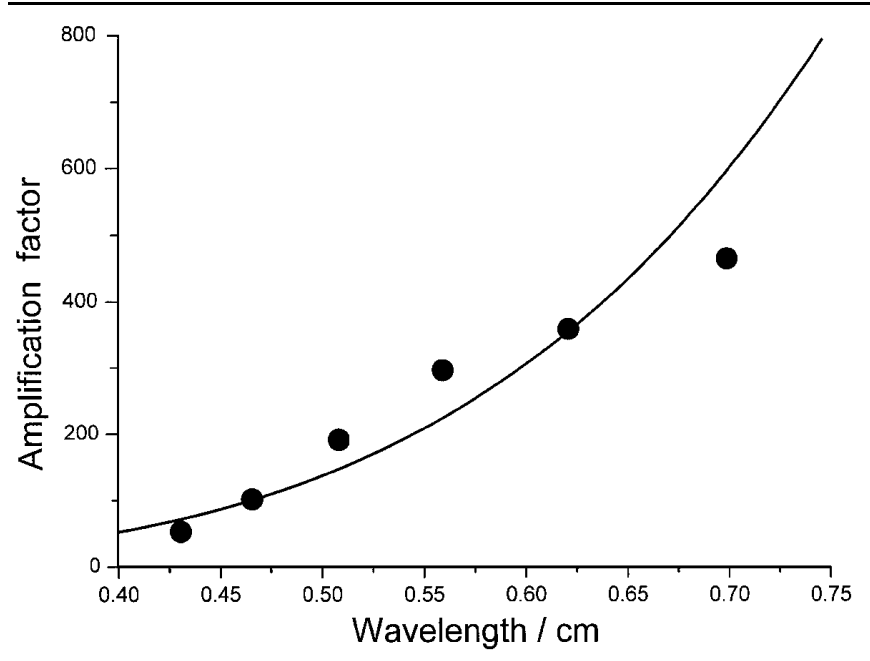

FIG. 4. The approximation amplification factors of transmission through a single metallic slit. The line is a simple $\lambda^{4}$ fit to the data.

From Fig. 2 we also note that the amplitude of the resonances decreases as the frequency is increased, leading to the amplification factor increase with wavelength shown in Fig. 4. This is, in part, caused by dividing the transmission by that of the empty aperture. According to standard aperture theory, this transmission should scale as $\lambda^{-4}$ [2]. So, as the wavelength increases, the transmission from the empty aperture decreases and the somewhat arbitrary transmission ratio increases. The curve shown in the data of Fig. 4 indicates that the data accord approximately to this form.

It is clear from the above results that the metal slits are acting as more than just resonant Fabry-Pérot-like cavities in which the coupled surface plasmons set up standing wave $[11,12]$. The transmitted intensity is orders of magnitude higher than the intensity arriving at the slit. In effect, the metal surface with a slit in it is behaving as a much wider device. This coupling effect, which may be in part like that mentioned in [8], allows incident energy arriving at the front face of the metallic geometry to flow into the slit. We may consider the slit as a high $Q$, narrow, resonant cavity for the amplification of the microwave transmission. The precise form of the metal surfaces either side of the slit has a strong impact upon the coupling of the radiation. In the experiment this takes the simple form of a pair of flat ended metal plates, and it may well be that introducing periodic structures on these faces will enhance coupling just as the periodicity of the hole arrays enhances coupling in the work of Ebbesen et al. [1]. It is certain from the modeling of this system that we have undertaken that the metallic character near the slit entrance and exit matter. This indicates, as for all the enhanced transmission work conducted so far, that surface plasmons at the entrance and exit faces of the resonant slits play an important role.
These results also have implications for experimental work on microwave materials and for some devices. For example, for applications involving voltage-controllable materials such as liquid crystals [13] the use of a small amount of sample is vital to obtain low voltage control and good alignment. The slit sample used in this Letter has indeed been subsequently filled with aligned liquid crystal to produce a tunable microwave device. Results of this work will be presented in a further publication.

In summary, the transmission of TM-polarized radiation through a single metallic slit, of width much less than wavelength, has been experimentally studied in the microwave region. Fabry-Pérot-like transmission features are recorded which confirm the theoretical predictions. For the experimental geometry realized, more than 2 orders of magnitude as much radiation is transmitted on resonance as compared with that directly incident on the slit. For this resonant transmission there has been no grating coupling, but, nevertheless, the metallic entrance and exit faces play a crucial role. One potential application derived from these results may be the use of very small amounts of material to control microwave propagation.

The authors thank the Engineering and Physical Sciences Research Council and QinetiQ (Farnborough) for their financial support.

[1] T. W. Ebbesen, H. J. Lezec, H.F. Ghaemi, T. Thio, and P. A. Wolff, Nature (London) 391, 667 (1998).

[2] H.A. Bethe, Phys. Rev. 66, 163 (1944).

[3] L. Martin-Moreno, F. J. García-Vidal, H. J. Lezec, K. M. Pellerin, T. Thio, J. B. Pendry, and T. W. Ebbesen, Phys. Rev. Lett. 86, 1114 (2001).

[4] U. Schröter and D. Heitmann, Phys. Rev. B 58, 15419 (1998).

[5] C. Sonnichsen, A. C. Durch, G. Steininger, M. Koch, G. von Plessen, and J. Feldmann, Appl. Phys. Lett. 76, 140 (2000).

[6] J. A. Porto, F. J. García-Vidal, and J. B. Pendry, Phys. Rev. Lett. 83, 2845 (1999).

[7] M. M. J. Treacy, Appl. Phys. Lett. 75, 606 (1999).

[8] S. Astilean, Ph. Lalanne, and M. Palamaru, Opt. Commun. 175, 265 (2000).

[9] Y. Takakura, Phys. Rev. Lett. 86, 5601 (2001).

[10] J. T. Adams and L. C. Botten, J. Opt. (France) 10, 109 (1979).

[11] M. B. Sobnack, W. C. Tan, N. P. Wanstall, T. W. Preist, and J. R. Sambles, Phys. Rev. Lett. 80, 5667 (1998).

[12] H. E. Went, A. P. Hibbins, J. R. Sambles, C. R. Lawrence, and A. P. Crick, Appl. Phys. Lett. 77, 2789 (2000).

[13] K. C. Lim, J. D. Margerum, A. M. Lackner, E. Sherman, M.-S. Ho, B. M. Fung, W. B. Genetti, and B. P. Grady, Mol. Cryst. Liq. Cryst. 302, 187 (1997). 